\title{
Nose Obstruction Symptom Evaluation Scale Outcomes in Covid 19 Patients in the Turkish Population and Prognostic Factors Affecting the Scale
}

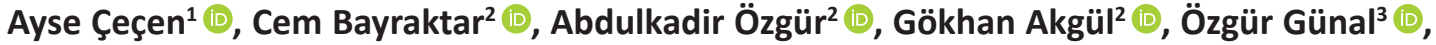 \\ Mehmet Eser Sancaktar ${ }^{2}$ (D), Merve Mutlu² ${ }^{(1)}$
}

\author{
${ }^{1}$ Samsun University, Faculty of Medicine, Department of Otolaryngology, Samsun, Turkey \\ ${ }^{2}$ Samsun Education and Research Hospital, Department of Otolaryngology, Samsun, Turkey \\ ${ }^{3}$ Samsun Education and Research Hospital, Department of Clinic of Infectious Diseases and Clinical Microbiology, Samsun, Turkey
}

ORCID ID: A.Ç. 0000-0001-6255-6125; C.B. 0000-0002-7446-9077; A.Ö. 0000-0002-6155-5988; G.A. 0000-0003-0699-6585;

Ö.G. 0000-0002-7744-4123; M.E.S. 0000-0002-1498-5539; M.M. 0000-0002-2532-1009

Citation: Cecen A, Bayraktar C, Ozgur A, Akgul G, Gunal O, Sancaktar MS, Mutlu M. Nose obstruction symptom evaluation scale outcomes in Covid 19 patients in the Turkish population and prognostic factors affecting the scale. Tr-ENT 2021;31(4):100-104.

https://doi.org/10.26650/Tr-ENT.2021.1001317

\section{ABSTRACT}

Objective: Coronavirus Disease 2019 (Covid 19) is a viral pandemic that emerged in East Asia and spread rapidly to the rest of the world and continues in our country. This study aims to evaluate the subjective severity of nasal obstruction with the Turkish Nasal Obstruction Symptom Evaluation (T-NOSE) in patients with laboratory-confirmed Covid 19 infection and to compare with the control group.

Material and Methods: One hundred fifty-seven patients with confirmed Covid 19 infection (group 1, n=157) and 91 individuals without Covid 19 infection as the control group (group 2, $n=91$ ) were included in the study. Nasal obstruction complaints of adult patients with Covid 19 infection were evaluated with the T-NOSE scale test and compared with the control group. Covid 19 patients were grouped according to the degree of thoracic $\mathrm{CT}$ involvement and the day that the NOSE test was performed.

Results: There was no statistically significant difference between the NOSE scale parameters of the Covid group and the control group ( $\mathrm{p}=0.19$ ). Also, Covid 19 patients were grouped according to the thoracic CT involvement degree and divided into three groups according to the day of the NOSE test. The relationship between these values, and NOSE was evaluated, and no statistically significant difference was found ( $p=0.65, p=0.385$ ). Conclusion: T-NOSE is a valid instrument with good internal consistency, reliability, and responsive instrument that is brief and easy to complete and has potential use for outcome studies in adults Covid 19 patients with nasal obstruction.

Keywords: NOSE, Turkish NOSE, Covid 19, Nasal obstruction, adult

\section{INTRODUCTION}

It is known that acute respiratory distress syndrome, which started in Wuhan city of Hubei region of China in December 2019, affected the whole world. Coronavirus disease 2019 (Covid 19) is an infectious respiratory disease caused by coronavirus 2 , a coronavirus family member, which has a high potential of human-to-human transmission (1).

An infected person may experience symptoms after an incubation period that can range from about 2 to 14 days (there have been rare cases of an incubation period of 29 days), while the person can still be contagious. Precautions such as careful personal hygiene, frequent hand washing, wearing masks and social distancing should be taken to limit the transmission (2).

Coronavirus mainly affects the lower respiratory tract and when the symptoms are examined, fever, fatigue, cough and dyspnea are at the forefront. However, anosmia-hyposmia, lack of taste, diarrhea, sore throat, headache, nasal congestion, rhinorrhea and skin lesions may also develop. In severe cases, pneumonia, acute respiratory distress syndrome, sepsis and septic shock, extending to patient's death, can occur $(3,4)$.

Corresponding Author: Ayşe Çeçen E-mail: aysebel55@hotmail.com

Submitted: 20.09.2021 • Accepted: 24.01.2022

This work is licensed under Creative Commons Attribution-NonCommercial 4.0 International License. 
In patients without pneumonia, the Covid 19 clinic is similar to some diseases frequently encountered by ENT physicians in the outpatient clinic. Cough, sore throat, shortness of breath, sputum production, fever, rhinorrhea, nasal congestion, oropharynx hyperemia and edema, lymphadenopathy and anosmia are among the ENT complaints and findings of Covid $19(5,6)$.

Nasal congestion is a common symptom with multifactorial origin, stated as a feeling of blockage or insufficient airflow through the nose by the patients. An otolaryngologist encounters this symptom during the examination of Covid 19 patients, as in other viral infections $(7,8)$.

The NOSE scale is a simple, commonly used, and well-validated quality of life tool specific to nasal airway obstruction. This scale determines the patient's subjective feelings (7).

The Nasal Obstruction Symptom Assessment (NOSE) scale was designed by Stewart et al. (9) in 2004 and has been validated in French (10), Portuguese (11), Italian (12) and Greek (13). Karahatay et al. It adapted the scale to Turkish in 2018 (7).

This study evaluates the subjective severity of nasal obstruction with the NOSE scale (T-NOSE) in patients with laboratoryconfirmed Covid 19 infection and to compare it with the control group.

\section{MATERIALS AND METHODS}

\section{Study design}

The study was conducted between May 2020 and September 2020, in Samsun Training and Research Hospital, by the Department of Otorhinolaryngology and Department of Infectious Diseases and Clinical Microbiology after approved by the SamsunTraining and Research Hospital Human Ethics Committee (Decision number: 2020/6/10). The study was conducted under the rules of the Helsinki Declaration. Patients hospitalized during this study and agreed to participate in the study were included in the study. The study was conducted on patients hospitalized in the Department of Infectious Diseases and Clinical Microbiology. One hundred fifty-seven patients with laboratory-confirmed Covid 19 infection (group 1, n=157) and 91 individuals without Covid 19 infection as the control group (group 2, $\mathrm{n}=91$ ) were included in the study. A written consent form was obtained from all patients.

\section{Patients}

Two hundred forty-eight cases, including 157 patients having Covid 19 (Covid Group) and 91 healthy controls (Control Group), were included in this prospective study.

Covid 19 infection diagnosis is based on serological tests with Covid 19 specific IgM or IgG and/or reverse transcriptasepolymerase chain reaction (RT-PCR).

Researchers applied current tests to patients with appropriate protective equipment to avoid viral transmission. Age, gender, accompanying systemic disease, smoking status, and nasal surgery history of all patients were questioned.
Those with nasal polyps, allergic rhinitis, chronic sinusitis, vasomotor rhinitis, sinonasal malignancy, significant septal deviation, conchal hypertrophy, trauma, or epistaxis history in the last $48 \mathrm{~h}$, who used drugs that may affect nasal obstruction such as systemic and local decongestants, local nasal steroids, anticholinergic agents in the last three months and patients who have had previous nasal surgery were excluded from the study.

All patients underwent anterior rhinoscopic examinations, and those with nasal pathologies such as sinusitis, septum deviation, and nasal polyposis that can cause nasal obstruction were also excluded from the study. A control group was formed from healthy individuals of appropriate age and gender for the patient group. All patients included in the study had NOSE tests.

The NOSE questionnaire, consisting of 5 questions about nasal obstruction experienced by the patients (nasal congestion or stuffiness, nasal blockage or obstruction, trouble breathing through the nose, trouble sleeping, unable to get enough air through) was validated by Stewart et al. (9). Each question is scored using a 5-point Likert scale, and the tool is then scaled to 0 to 100 total points, multiplying the raw score by 5 . Higher NOSE survey scores correspond to severe nasal congestion. The reliability and consistency of this scale make it useful to understand how nasal obstruction affects the patient's quality of life. All participants completed the questionnaire themselves without assistance. The NOSE scores of the Covid group were compared with the scores of the control group.

Routine thoracic computed tomography (CT) of Covid 19 patients were grouped according to the normal, focal, and diffuse involvement, and the relationship between these groups and NOSE tests was evaluated. Patients without pulmonary involvement were accepted as normal, those limited to one lung lobe as focal and those with widespread involvement in both lungs as diffuse.

The patients were divided into three groups (group: $1,1^{\text {st }} 3^{\text {rd }}$ days, group 2: $4^{\text {th }}-7^{\text {th }}$ days, group 3: $8^{\text {th }}-14^{\text {th }}$ days) according to the day of the NOSE test, and the relationship between these groups and NOSE scale was evaluated.

\section{Statistical methods}

Statistical Package for Social Science (SPSS) version 21.0 (SPSS Inc., Chicago, IL, and USA) was used for statistical analysis. In data assessment, Kolmogrof -Siminorv was used to test whether data normally distributed or not. The Kruskal-Wallis test was used as a non-parametric test to compare the groups. Chi-square test was used to assess the intergroup difference between groups in gender. Significance level was taken as 0.05 .

\section{RESULTS}

Two hundred forty-eight cases, including 157 Covid 19 patients (group 1: 71 females and 86 males) (Covid Group) and 91 healthy controls (group 2: 44 females and 47 males) (Control Group), were included in this prospective study. The mean age of Covid (group 1) and control groups (group 2) were 
$45.80 \pm 16.84$ and $46.62 \pm 15.35$ years, respectively. The groups were similar in terms of age and gender $\left(p=0.45, p=0.63^{\Psi}\right)$ (Table 1).

Table 1: The Results of NOSE Test in Patients with Covid 19 and Healthy Controls and Demographic Features

\begin{tabular}{lccc}
\hline & $\begin{array}{c}\text { Covid group } \\
(\mathbf{n}=157)\end{array}$ & $\begin{array}{c}\text { Control group } \\
(\mathbf{n}=91)\end{array}$ & $\mathbf{p}^{\mathrm{r}}$ value \\
\hline Gender & & & \\
$\quad$ Male & 86 & 47 & $0.63^{\Psi}$ \\
$\quad$ Female & 71 & 44 & \\
Age & $45.80 \pm 16.84$ & $46.62 \pm 15.35$ & 0.45 \\
NOSE questionnaire & $0.82 \pm 1.26$ & $0.59 \pm 1.06$ & 0.19 \\
\hline
\end{tabular}

Y: Mann-Whitney U test, $\Psi$ : Chi-square test

The mean scores for the NOSE test were determined as $0.82 \pm 1.26$ for group 1 and $0.59 \pm 1.06$ for group 2 . There was no statistically significant difference between the groups in terms of NOSE tests $(p=0.19)$ (Table 1$)$.

Covid 19 patients were grouped according to the degree of thoracic CT involvement. The relationship between these groups and NOSE tests was evaluated, and the result was not statistically significant ( $p=0.65$ ) (Table 2 ).

Table 2: Correlation table between NOSE test and the degree of thoracic CT involvement

\begin{tabular}{lcc}
\hline CT involvement type (n) & NOSE score & p value $^{*}$ \\
\hline Normal (95) & $0.81 \pm 1.33$ & \\
Focal (12) & $0.66 \pm 1.23$ & 0.65 \\
Diffuse (50) & $0.88 \pm 1.15$ & \\
\hline
\end{tabular}

*Kruskal Wallis test

The patients were also divided into three groups according to the day the NOSE test was performed. The relationship between these values and NOSES was evaluated, and no statistically significant difference was found ( $p=0.385$ ) (Table 3).

Table 3: Comparisons between NOSE test and days

\begin{tabular}{lcc}
\hline Days $(n)$ & NOSE score & p value $^{*}$ \\
\hline $1^{\text {st }}-3^{\text {rd }}$ days $(n=50)$ & $0.880 \pm 1.533$ & 0.385 \\
$4^{\text {th }}-7^{\text {th }}$ days $(n=99)$ & $1.656 \pm 2.839$ & \\
$8^{\text {th }}-14^{\text {th }}$ days $(n=8)$ & $1.500 \pm 1.603$ & \\
\hline
\end{tabular}

*Kruskal Wallis test

\section{DISCUSSION}

SARS-CoV-2, the agent of Covid 19, is a sub-type of the coronavirus family defined in the 1960 s. Coronaviruses are a family of viruses that can cause respiratory and gastrointestinal infections in animals and humans, settle in the lower respiratory tract, cause pneumonia, and then death due to respiratory failure. These viruses are animal diseases (zoonoses), but they can infect humans and cause epidemics via mutations (14).

The clinical picture of SARS-CoV-2 infection can be asymptomatic, mild, severe, or fatal. According to China's data, $80 \%$ of Covid 19 cases are relatively mild, while $15 \%$ of patients have severe clinical course. However, more than $80 \%$ of the cases are asymptomatic and cannot be identified since they are not tested (6).

The complaints of Covid 19 patients are nonspecific like other respiratory infections. Fever (43\%-98\%), cough (68\%-82\%), fatigue (38-44\%), sore throat (13.9-17.4\%), dry cough (59.4\%) and sputum (28-33\%) can be seen. Publications have reported that nearly half of the patients have a loss of smell and taste. Nasal obstruction (4.8\%) and rhinorrhea (4\%) have been reported to be very rare, unlike other upper respiratory tract infections (8).

According to Krajewska et al. review, fever, fatigue, and dry cough are considered the most common symptoms of Covid 19. Anorexia, shortness of breath, sputum production, and myalgia have been reported in more than $25 \%$ of cases. Sore throat, rhinorrhea, headache, nausea, and diarrhea are less common symptoms that are present in mild or moderate disease forms (15).

In a meta-analysis of Lovato et al., when evaluated in terms of otolaryngology symptoms, they reported that sore throat (12.4\%), nasal congestion (3.7\%), and rhinorrhea were the most common symptoms among patients with Covid 19 (16).

Covid 19 can be confused with the flu or common cold regarding initial symptoms, causing patients to apply to the otorhinolaryngology clinic in the foreground. In patients without pneumonia, the clinical presentation of Covid 19 is similar to some diseases that ENT physicians frequently encounter in the outpatient clinic. While the rhinorrhea is more common in colds than other diseases, dry cough is prominent in influenza and Sars-CoV-2 infection. Sudden onset symptoms are more compatible with Covid 19 (5).

In the literature, the rates of nasal symptoms accompanying Covid 19 vary (nasal congestion: 3.7\%-67.8\%, rhinorrhea: 4\%-60.1\%). Therefore, we planned to perform the NOSE questionnaire in Covid 19 patients $(5,6,8,15-17)$.

Similar to the literature, the most common symptoms in our patients were fever $(22.9 \%, n=36)$, fatigue $(19.1 \%, n=30)$, cough $(16.5 \%, n=26)$, and dyspnea $(12.7 \%, n=20)$. Loss of taste and smell $(10.1 \%), n=16$, nasal congestion $(7.6 \%, n=12)$, rhinorrhea $(6.3 \%, n=10)$, and sore throat $(4.4 \%, n=7)$ are among the rare upper respiratory tract symptoms we observed in our patients.

Nasal airway obstruction (NAO) is one of the most common clinical indications for otolaryngology referral. The estimated economic burden of this symptom is more than $\$ 5$ billion per year (18). Numerous studies have tried improving this condition's diagnosis. However, NAO remains a diagnostic 
challenge due to inconsistencies between subjective symptoms and objective findings such as physical examination, rhinomanometry, peak nasal inspiratory flow, acoustic rhinometry, or radiographic findings $(18,19)$.

The consensus has shifted toward weighing subjective assessments more due to inconsistencies between objective measurements and subjective evaluations (20). There has been a trend toward using patient-reported outcome measures to assess nasal obstruction, particularly in the form of the NOSE scale. The NOSE scale is a questionnaire used to evaluate patients' quality of life with nasal congestion $(9,20,21)$.

In the literature, the NOSE scale has been used in many studies to evaluate the quality of life in patients with nasal obstruction. The NOSE scale has been the most structurally relevant diseasespecific quality of life tool that was developed to assess nasal congestion, and it has evidence supporting its validity, reliability and sensitivity $(13,21,22)$.

Based on this information in the literature, we evaluated the subjective severity of nasal obstruction with the NOSE scale (T-NOSE) to evaluate nasal symptoms in Covid 19 patients, compared with the control group. In accordance with the literature, we observed that the complaints of nasal obstruction in Covid 19 disease are among the rare upper respiratory tract symptoms in our patients.

Due to high virus concentrations in the nasal cavity, nasopharynx, and oropharynx and close contact of otolaryngologists with the upper respiratory mucosa of the patients, the highest nosocomial transmission rates were reported among otolaryngologists $(8,23)$.

This study is also critical since it is the first study to investigate NOSE scale changes in patients with Covid 19 in the adult population and compare them with healthy individuals.

One of the crucial limitations of our study is the relatively small number of patients. We think that studies with a larger sample size may contribute to further knowledge.

We applied the NOSE questionnaire to Covid 19 patients who were hospitalized in the Covid inpatient clinic. It is thought that nasal symptoms of outpatients are more common than inpatients. Comparing the test with outpatients and inpatients would have changed the results of our study (17).

Covid 19 is a disease with increasing importance in many countries, including Turkey.

We think that the Covid 19 does not significantly change the NOSE tests, which aims to evaluate the upper respiratory tract activity since it dominantly affects the lower respiratory tract.

Since specific otolaryngological symptoms such as nasal obstruction and rhinorrhea are rare in Covid 19 disease, unlike other upper respiratory tract infections, otolaryngologists should consider every patient as positive.
Ethics Committee Approval: The study was conducted between May 2020 and September 2020, in SamsunTraining and Research Hospital, by the Department of Otorhinolaryngology and Department of Infectious Diseases and Clinical Microbiology after approved by the SamsunTraining and Research Hospital Human Ethics Committee (Decision number: 2020/6/10). The study was conducted under the rules of the Helsinki Declaration.

Informed Consent: Written informed consent was obtained.

Peer-Review: Externally peer-reviewed.

Author Contributions: Conception/Design of Study- A.Ç., C.B., A.Ö., G.A., Ö.G., M.E.S., M.M.; Data Acquisition- G.A., M.B.; Data Analysis/ Interpretation- A.Ç., C.B., A.Ö., G.A., Ö.G., M.E.S., M.M.; Drafting Manuscript- A.Ç., C.B., A.Ö., G.A., Ö.G., M.E.S., M.M.; Critical Revision of Manuscript- A.Ç., C.B., A.Ö., G.A., Ö.G., M.E.S., M.M.; Final Approval and Accountability- A.Ç., C.B., A.Ö., G.A., Ö.G., M.E.S., M.M.

Conflict of Interest: Authors declared no conflict of interest.

Financial Disclosure: Authors declared no financial support.

\section{REFERENCES}

1. Ozen $\mathrm{G}, \mathrm{Koc} H$, Aksoy $\mathrm{C}$. Health anxiety status of elite athletes in COVID-19 social isolation period. Bratisl Med J 2020;121(12): 88893.

2. Fiorillo L, Cervino G, Matarese M, D'Amico Cesare, Surace Giovanni, Paduano Valeria, et al. COVID-19 Surface Persistence: A Recent Data Summary and Its Importance for Medical and Dental Settings. Int J Environ Res Public Health 2020;17(9):3132.

3. Zhang Y, Xu J, Li H, Cao B. A novel coronavirus (COVID-19) outbreak: A call for action. Chest 2020;157(4):e99-e101.

4. Yang Y, Shang W, Rao X. Facing the COVID-19 outbreak: What should we know and what could we do? J Med Virol 2020;92(6):536-7.

5. Guan WJ, Ni ZY, Hu Y, Liang WH, Ou CQ, He JX, et al. Clinical Characteristics of Coronavirus Disease 2019 in China. N Engl J Med 2020;382(18):1708-20.

6. Huang C, Wang Y, Li X, Ren L, Zhao J, Hu Y, et al. Clinical features of patients infected with 2019 novel coronavirus in Wuhan, China. Lancet 2020;395(10223):497-506.

7. Karahatay S, Taşlı H, Karakoç Ö, Aydın Ü, Türker T. Reliability and validity of the Turkish Nose Obstruction Symptom Evaluation (NOSE) scale. Turk J Med Sci 2018;48:212-6.

8. Vukkadala N, Qian ZJ, Holsinger FC, Patel ZM, Rosenthal E. COVID-19 and the Otolaryngologist: Preliminary Evidence-Based Review. Laryngoscope 2020;130(11):2537-43.

9. Stewart MG, Witsell DL, Smith TL, Weaver EM, Yueh B, Hannley MT. Development and validation of the Nasal Obstruction Symptom Evaluation (NOSE) scale. Otolaryngol Head Neck Surg 2004;130(2):157-63.

10. Marro M, Mondina M, Stoll D, de Gabory L. French validation of the NOSE and Rhino QOL questionnaires in the management of nasal obstruction. Otolaryngol Head Neck Surg 2011;144(6):988-93.

11. Bezerra TF, Padua FG, Pilan RR, Stewart MG, Voegels RL. Crosscultural adaptation and validation of a quality of life questionnaire: the Nasal Obstruction Symptom Evaluation questionnaire. Rhinology 2011;49(2):227-31. 
12. Mozzanica F, Urbani E, Atac M, Scotta G, Luciano K, Bulgheroni $C$, et al. Reliability and validity of the Italian Nose Obstruction Symptom Evaluation (I-NOSE) scale. Eur Arch Otorhinolaryngol 2013;270(12):3087-94.

13. Lachanas VA, Tsiouvaka S, Tsea M, Hajiioannou JK, Skoulakis CE. Validation of the Nasal Obstruction Symptom Evaluation (NOSE) scale for Greek patients. Otolaryngol Head Neck Surg 2014;151(5):819-23.

14. Channappanavar R, Perlman S. Pathogenic human coronavirus infections: causes and consequences of cytokinestorm and immunopathology. Semin Immunopathol 2017;39(5):529-39.

15. Krajewska J, Krajewski W, Zub K, Zatoński T. COVID-19 in otolaryngologist practice: a review of current knowledge. Eur Arch Otorhinolaryngol 2020;277(7):1885-97.

16. Lovato A, de Filippis C. Clinical Presentation of COVID-19: A Systematic Review Focusing on Upper Airway Symptoms. Ear Nose Throat J 2020;99(9):569-76.

17. Lechien JR, Chiesa-Estomba CM, Place S, Van Laethem Y, Cabaraux $P, M a t Q$, et al. Clinical and epidemiological characteristics of 1420 European patients with mild-to-moderate coronavirus disease 2019. J Intern Med 2020;288(3):335-44.
18. Casey KP, Borojeni AAT, Koenig LJ, Rhee JS, Garcia GJM. Correlation between subjective nasal patency and intranasal airflow distribution. Otolaryngol Head Neck Surg 2017;156 (4):741-50.

19. Lam DJ, James KT, Weaver EM. Comparison of anatomic, physiological, and subjective measures of the nasalairway. Am J Rhinol 2006;20(5):463-70.

20. Cannon DE, Rhee JS. Evidence-Based Practice: Functional Rhinoplasty. Otolaryngol Clin North Am 2012;45(5):1033-43.

21. Gu JT, Kaplan S, Greenfield S, Calloway H, Wong BJF. Validation of a Septoplasty Deformity Grading System for the Evaluation of Nasal Obstruction. Laryngoscope 2019;129(3):586-93.

22. Barone M, Cogliandro A, Di Stefano N, Tambone V, Persichetti P. A Systematic review of patient-reported outcome measures after rhinoplasty. Eur Arch Otorhinolaryngol 2016;274(4):1807-11.

23. Lu R, Zhao X, Li J, Niu P, Yang P, Wu H, et al. Genomic characterisation and epidemiology of 2019 novel coronavirus: implications for virus origins and receptor binding. Lancet 2020;395(10224):565-74. 